\title{
Suppression Measured from Chinchilla Auditory-Nerve-Fiber Responses Following Noise-Induced Hearing Loss: Adaptive-Tracking and Systems-Identification Approaches
}

\author{
Mark Sayles, Michael K. Walls and Michael G. Heinz
}

\begin{abstract}
The compressive nonlinearity of cochlear signal transduction, reflecting outer-hair-cell function, manifests as suppressive spectral interactions; e.g., twotone suppression. Moreover, for broadband sounds, there are multiple interactions between frequency components. These frequency-dependent nonlinearities are important for neural coding of complex sounds, such as speech. Acoustic-traumainduced outer-hair-cell damage is associated with loss of nonlinearity, which auditory prostheses attempt to restore with, e.g., "multi-channel dynamic compression" algorithms.
\end{abstract}

Neurophysiological data on suppression in hearing-impaired (HI) mammals are limited. We present data on firing-rate suppression measured in auditory-nerve-fiber responses in a chinchilla model of noise-induced hearing loss, and in normalhearing $(\mathrm{NH})$ controls at equal sensation level. Hearing-impaired (HI) animals had elevated single-fiber excitatory thresholds (by $\sim 20-40 \mathrm{~dB}$ ), broadened frequency tuning, and reduced-magnitude distortion-product otoacoustic emissions; consistent with mixed inner- and outer-hair-cell pathology. We characterized suppression using two approaches: adaptive tracking of two-tone-suppression threshold ( $62 \mathrm{NH}$, and $35 \mathrm{HI}$ fibers), and Wiener-kernel analyses of responses to broadband noise (91 $\mathrm{NH}$, and $148 \mathrm{HI}$ fibers). Suppression-threshold tuning curves showed sensitive low-

M. G. Heinz $(\bowtie) \cdot$ M. Sayles $\cdot$ M. K. Walls

Department of Speech, Language, \& Hearing Sciences, Purdue University, Lyles-Porter Hall, 715 Clinic Drive, West Lafayette, IN 47907-2122, USA

e-mail: mheinz@purdue.edu

M. G. Heinz

Weldon School of Biomedical Engineering, Purdue University, 206 S. Martin Jischke Drive, West Lafayette, IN 47907-2032, USA

M. Sayles

Laboratory of Auditory Neurophysiology, Katholieke Universiteit Leuven, Campus

Gasthuisberg-O\&N II, Herestraat 49-bus 1021, 3000 Leuven, Belgium

e-mail: sayles.m@gmail.com

M. K. Walls

e-mail: mkwalls@purdue.edu

(C) The Author(s) 2016

P. van Dijk et al. (eds.), Physiology, Psychoacoustics and Cognition in Normal and Impaired Hearing, Advances in Experimental Medicine and Biology 894,

DOI 10.1007/978-3-319-25474-6_30 
side suppression for $\mathrm{NH}$ and $\mathrm{HI}$ animals. High-side suppression thresholds were elevated in HI animals, to the same extent as excitatory thresholds. We factored second-order Wiener-kernels into excitatory and suppressive sub-kernels to quantify the relative strength of suppression. We found a small decrease in suppression in HI fibers, which correlated with broadened tuning. These data will help guide novel amplification strategies, particularly for complex listening situations (e.g., speech in noise), in which current hearing aids struggle to restore intelligibility.

Keywords Auditory nerve - Suppression - Frequency-dependent nonlinearity • Chinchilla - Spike-triggered neural characterization - Wiener kernel - Singularvalue decomposition - Threshold tracking · Frequency tuning · Hearing impairment - Noise exposure $\cdot$ Acoustic trauma $\cdot$ Cochlear hearing loss $\cdot$ Auditory-brain-stem response $\cdot$ Otoacoustic emissions

\section{Introduction}

Frequency-dependent cochlear signal-transduction nonlinearities manifest as suppressive interactions between acoustic-stimulus components (Sachs and Kiang 1968; de Boer and Nuttall 2002; Versteegh and van der Heijden 2012, 2013). The underlying mechanism is thought to be saturation of outer-hair-cell receptor currents (Geisler et al. 1990; Cooper 1996). Despite the importance of suppression for neural coding of complex sounds (e.g., Sachs and Young 1980), relatively little is known about suppression in listeners with cochlear hearing loss (Schmiedt et al. 1990; Miller et al. 1997; Hicks and Bacon 1999). Here we present preliminary data on firing-rate suppression, measured with tones, and with broadband noise, from ANFs in chinchillas (Chinchilla laniger), following noise-induced hearing loss. Quantitative descriptions of suppression in the hearing-impaired auditory periphery will help guide novel amplification strategies, particularly for complex listening situations (e.g., speech in noise), in which current hearing aids struggle to improve speech intelligibility.

\section{Methods}

\subsection{Animal Model}

Animal procedures were under anesthesia, approved by Purdue University's IACUC, and followed NIH-issued guidance. Evoked-potential and DPOAE measures, and noise exposures, were with ketamine $(40 \mathrm{mg} / \mathrm{kg}$ i.p. $)$ and xylazine $(4 \mathrm{mg} / \mathrm{kg}$ s.c.). Single-unit neurophysiology was done with sodium pentobarbital (boluses: 5-10 mg/hr. i.v.). 


\subsubsection{Noise Exposure \& Hearing-Loss Characterization}

Two groups of chinchillas are included: normal-hearing controls (NH), and hearingimpaired (HI) animals with a stable, permanent, sensorineural hearing loss. Prior to noise exposure, we recorded auditory brainstem responses (ABRs) and distortionproduct otoacoustic emissions (DPOAEs) from the HI group, verifying "normal" baseline status.

ABRs were recorded in response to pure-tone bursts, at octave-spaced frequencies between 0.5 and $16 \mathrm{kHz}$. Threshold was determined according to statistical criteria (Henry et al. 2011). DPOAE stimulus primaries $\left(f_{1}\right.$ and $\left.f_{2}\right)$ were presented with equal amplitude (75-dB SPL), and with a constant $f_{2} / f_{1}$ ratio of 1.2. $f_{2}$ varied between 0.5 and $12 \mathrm{kHz}$, in 2-semitone steps.

Animals were exposed to either a 500-Hz-centered octave-band noise at 116-dB SPL for $2 \mathrm{~h}$, or a 2-kHz-centered 50-Hz-wide noise at 114-115-dB SPL for $4 \mathrm{~h}$. They were allowed to recover for 3-4 weeks before an acute single-unit experiment. At surgery, ABR and DPOAE measures were repeated prior to any additional intervention (for NH animals, these were their only ABR and DPOAE measurements).

\subsubsection{Single-Unit Neurophysiology}

The auditory nerve was approached via a posterior-fossa craniotomy. ANFs were isolated using glass pipettes with 15-25 M $\Omega$ impedance. Spike times were recorded with $10-\mu$ s resolution. Stimulus presentation and data acquisition were controlled by MATLAB programs interfaced with hardware modules (TDT and National Instruments). For HI animals, ANF characteristic frequency (CF) was determined by the high-side-slope method of Liberman (1984).

\subsection{Stimuli}

\subsubsection{Adaptive-Tracking: Suppression-Threshold Tuning}

This two-tone suppression (2TS) adaptive-tracking technique is based on Delgutte (1990). Stimuli were sequences of 60-ms duration supra-threshold tones at CF, with or without a second tone at the suppressor frequency (FS; Fig. 1). For each FS frequency-level combination, 10 repetitions of the two-interval sequence were presented. The decision to increase or decrease sound level was made based on the mean of the 2 nd through 10th of these two-interval comparisons. The algorithm sought the lowest sound level of FS which reduced the response to the CF tone by

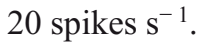



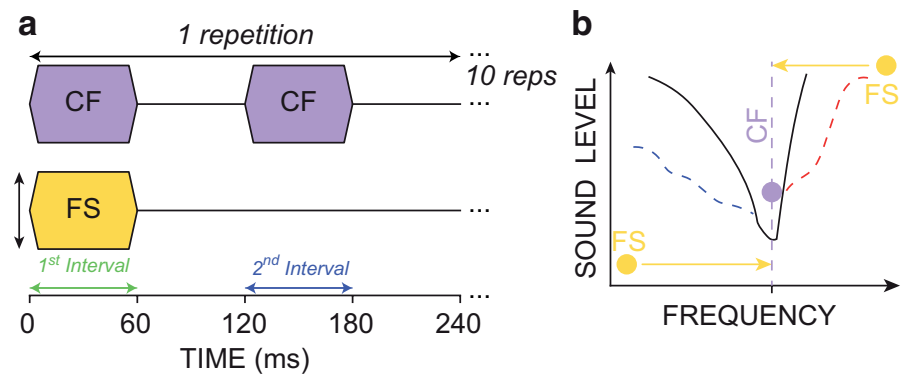

Fig. 1 Schematized stimulus paradigm: adaptive tracking. (B) Black line, excitatory tuning curve; Red and blue dashed lines, high- and low-side suppression-threshold curves, respectively

\subsubsection{Systems-Identification Approach}

Using spike-triggered characterization, we probed suppressive influences on firing rate in response to broadband-noise stimulation (e.g., Lewis and van Dijk 2004; Schwartz et al. 2006). Our implementation is based on singular-value decomposition of the second-order Wiener kernel $\left(h_{2}\right)$ in response to $16.5-\mathrm{kHz}$ bandwidth, 15dB SL noise (Lewis et al. 2002a, 2002b; Recio-Spinoso et al. 2005). We collected $\sim 10-20 \mathrm{~K}$ spike times per fiber.

\subsection{Analyses}

\subsubsection{Second-Order Wiener Kernels}

For each fiber, $h_{2}$ was computed from the second-order cross-correlation between the noise-stimulus waveform $x(t)$ and $N$ spike times. The spike-triggered cross correlation was sampled at $50 \mathrm{kHz}$, and with maximum time lag $\tau$ of $10.2 \mathrm{~ms}$ ( $m=512$ points) for $\mathrm{CFs}>3 \mathrm{kHz}$, or $20.4 \mathrm{~ms}$ ( $m=1024$ points) for CFs $<3 \mathrm{kHz} . h_{2}\left(\tau_{1}, \tau_{2}\right)$ is calculated as

$$
\frac{N_{0}}{A^{2}}\left[R_{2}\left(\tau_{1}, \tau_{2}\right)-\phi_{x x}\left(\tau_{2}-\tau_{1}\right)\right]
$$

where $\tau_{1}$ and $\tau_{2}$ are time lags, $A$ the instantaneous noise power, $N_{0}$ the mean firing rate in spikes s ${ }^{-1}$, and $R_{2}\left(\tau_{1}, \tau_{2}\right)$ the second-order reverse-correlation function calculated as

$$
\frac{1}{N} \sum_{i=1}^{N} x\left(t_{i}-\tau_{1}\right) x\left(t_{i}-\tau_{2}\right)
$$


with $t_{i}$ the $i^{\text {th }}$ spike time and $\phi_{x x}\left(\tau_{2}-\tau_{1}\right)$ the stimulus autocorrelation matrix. So computed, $h_{2}$ is an $m$-by- $m$ matrix with units of spikes $\cdot \mathrm{s}^{-1} \cdot \mathrm{Pa}^{-2}$ (Recio-Spinoso et al. 2005). We used singular-value decomposition to parse $h_{2}$ into excitatory $\left(h_{2 \varepsilon}\right)$ and suppressive $\left(h_{2 \sigma}\right)$ sub-kernels (Lewis et al. 2002a, 2002b; Lewis and van Dijk 2004; Rust et al. 2005; Sneary and Lewis 2007).

\subsubsection{Excitatory and Suppressive Sub-Kernels}

Using the MATLAB function $s v d, h_{2} \mathrm{~s}$ were decomposed as

$$
h_{2}=\mathrm{USV}
$$

where $\mathrm{U}, \mathrm{S}$ and $\mathrm{V}$ are $m$-by- $m$ matrices. The columns of $\mathrm{U}$ and rows of $\mathrm{V}$ are the left and right singular vectors, respectively. $\mathrm{S}$ is a diagonal matrix, the nonzero values of which are the weights of the corresponding-rank vectors. The decomposition can be rephrased as

$$
h_{2}=\sum_{j=1}^{m} k_{j} u_{j} v_{j}
$$

where $u_{j}$ and $v_{j}$ are column vector elements of $\mathrm{U}$ and $\mathrm{V}$, respectively, and $k_{j}$ is the signed weight calculated as

$$
k_{j}=\operatorname{sgn}\left[u_{j}(j) v_{j}(j)\right] s_{j}
$$

where $s g n$ is the signum function, $u_{j}(j)$ is the $j^{\text {th }}$ element of the $j^{\text {th }}$ left singular vector, $v_{j}(j)$ is the $j^{\text {th }}$ element of the $j^{\text {th }}$ right singular vector, and $s_{j}$ is the $j^{\text {th }}$ element of the nonzero diagonal of $\mathrm{S}$.

Positively and negatively weighted vectors are interpreted as excitatory and suppressive influences, respectively (Rust et al. 2005; Schwartz et al. 2006; Sneary and Lewis 2007). However, mechanical suppression, adaptation, and refractory effects may all contribute to what we term "suppressive" influences.

To determine statistical significance of each weighted vector, we re-computed $h_{2}$ from 20 different spike:train randomizations, conserving the first-order inter-spikeinterval distribution. Based on this bootstrap distribution, weights were expressed as $z$-scores, and any vector with $|z|>3$ and rank $\leq 20$ was considered significant. We calculated a normalized excitatory-suppressive ratio as

$$
R_{\varepsilon, \sigma}=\frac{\sum_{j=1}^{N_{\varepsilon}}\left|Z_{j \varepsilon}\right|-\sum_{j=1}^{N_{\sigma}}\left|Z_{j \sigma}\right|}{\sum_{j=1}^{N_{\varepsilon}}\left|Z_{j \varepsilon}\right|+\sum_{j=1}^{N_{\sigma}}\left|Z_{j \sigma}\right|}
$$

for $N_{\varepsilon}$ significant excitatory vectors and $N_{\sigma}$ significant suppressive vectors. This normalized ratio varies from 1 (only excitation, no suppression), through 0 (equal 
excitation and suppression), to -1 (only suppression and no excitation: in practice $R_{\mathcal{\varepsilon}, \sigma}<0$ does not occur).

\subsubsection{Spectro-Temporal Receptive Fields}

Based on Lewis and van Dijk (2004), and Sneary and Lewis (2007), we estimated the spectro-temporal receptive field (STRF) from $h_{2}$. These STRFs indicate the timing of spectral components of the broadband-noise stimulus driving either increases or decreases in spike rate. Moreover, the STRF calculated from the whole $h_{2}$ kernel is the sum of excitatory and suppressive influences. Therefore, we also determined STRFs separately from $h_{2 \varepsilon}$ and $h_{2 \sigma}$ to assess the tuning of excitation and suppression (Sneary and Lewis 2007).

\section{Results}

\subsection{Hearing-Loss Characterization}

Noise exposure elevated ABR threshold, and reduced DPOAE magnitude, across the audiogram; indicating a mixed inner- and outer-hair-cell pathology (Fig. 2).
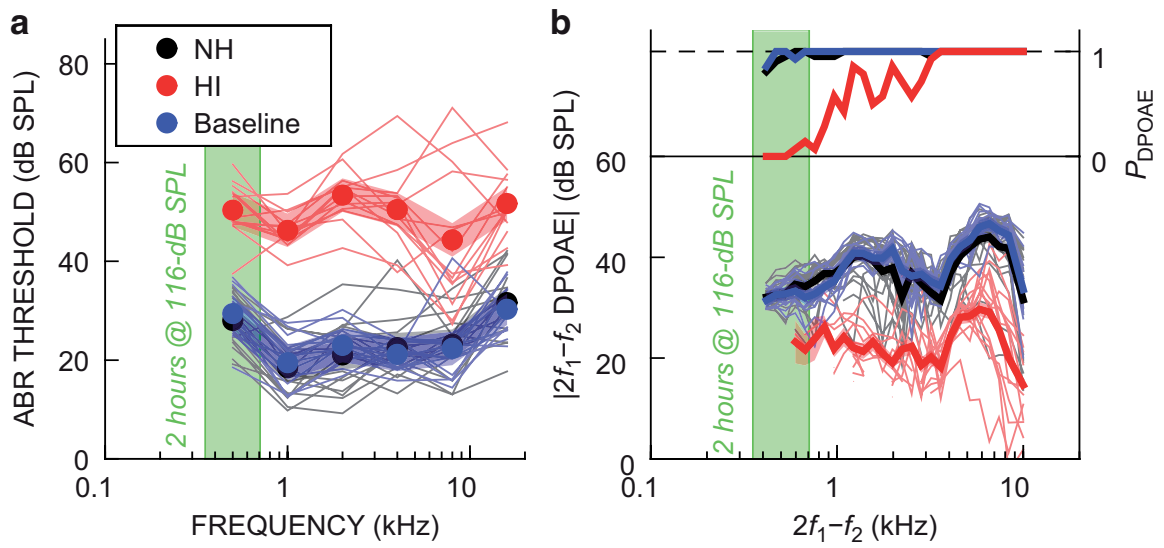

Fig. 2 Audiometric characterization. a ABR thresholds. Thin lines, individual-animal data; symbols, within-group least-squares-mean values; shading, S.E.M; green area, noise-exposure band. b Lower plot, DPOAE magnitude; Upper plot, probability of observing a DPOAE above the noise floor; thick lines, within-group least-squares means; shading, S.E.M 


\subsection{Suppression Threshold}

Two-tone-suppression tuning curves were obtained from $62 \mathrm{NH}$ fibers, and $35 \mathrm{HI}$ fibers (Fig. 3a and b). HI excitatory tuning curves show threshold elevation and broadened tuning (Fig. 3c). For NH fibers, high-side 2TS was always observed (Fig. 3a). However, in 6 of $35 \mathrm{HI}$ fibers, we could not detect significant high-side 2TS (Fig. 3b). These fibers have very broadened excitatory tuning (Fig. 3c). In HI fibers with detectable high-side $2 \mathrm{TS}$, the $\mathrm{dB}$ difference between excitatory threshold and suppressive threshold was not greater than observed in NH fibers (Fig. 3d). For many fibers high-side-2TS threshold was within $20 \mathrm{~dB}$ of on-CF excitatory threshold. Low-side 2TS-threshold estimates are surprisingly low (Fig. 3a and b). All fibers had low-side suppressive regions, regardless of hearing status, typically in the region of 0 - to $20-\mathrm{dB}$ SPL.
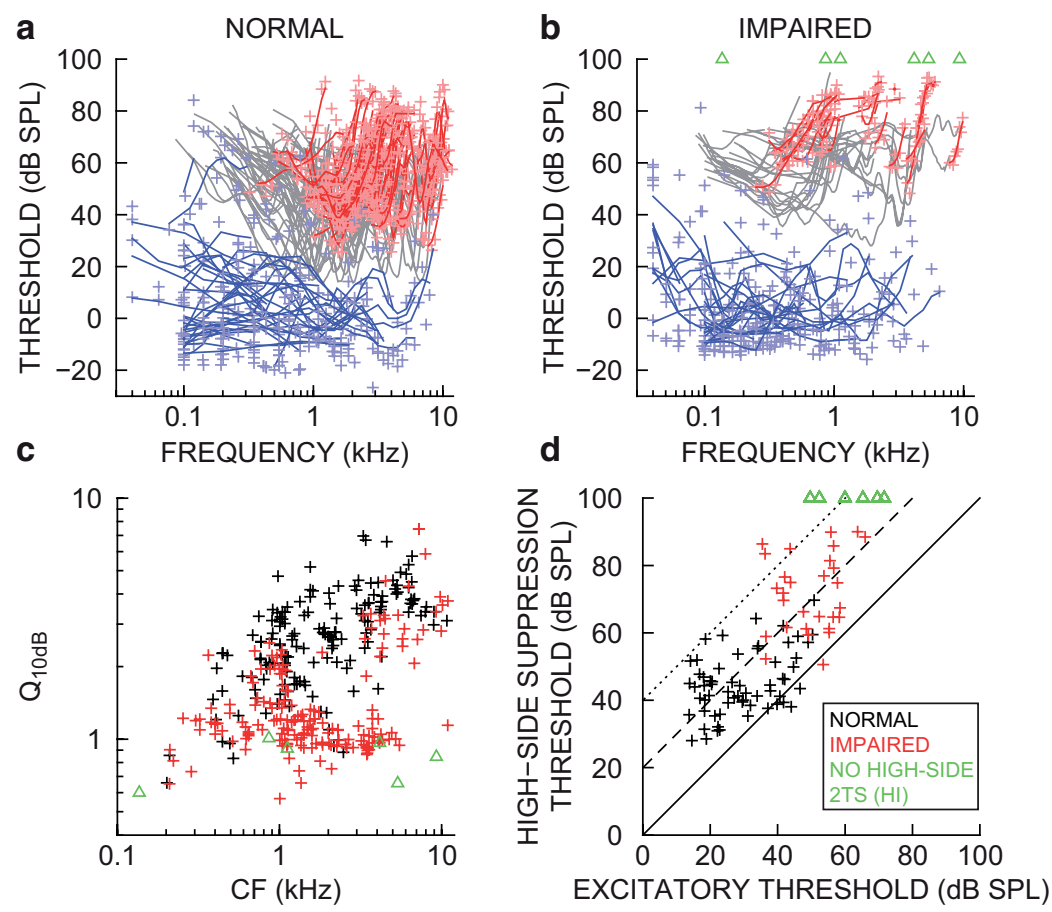

Fig. 3 Two-tone-suppression threshold. a NH: gray, excitatory-threshold tuning curves; red lines (fits) and crosses (data), high-side suppression-threshold tuning curves; blue lines and crosses, low-side suppression-threshold. b, HI data. c $Q_{10 \mathrm{~dB}}$. d Solid line, equality; dashed and dotted lines, 20- and 40-dB shifts in suppressive threshold, respectively. b-d, Green triangles at 100-dB SPL, $\mathrm{CFs}$ of $\mathrm{HI}$ fibers with no high-sided suppression 


\subsection{Wiener-Kernel Estimates of Suppression}

Figure 4 shows second-order Wiener-kernel and STRF analyses from the responses of a single medium-spontaneous-rate $\left(<18\right.$ spikes s$\left.^{-1}\right)$ ANF, with $\mathrm{CF} 4.2 \mathrm{kHz}$, recorded in a NH chinchilla. The $h_{2}$ kernel is characterized by a series of parallel diagonal lines representing the non-linear interactions driving the ANF's response to noise (Fig. 4a; Recio-Spinoso et al. 2005). The STRF derived from $h_{2}$ shows sup-
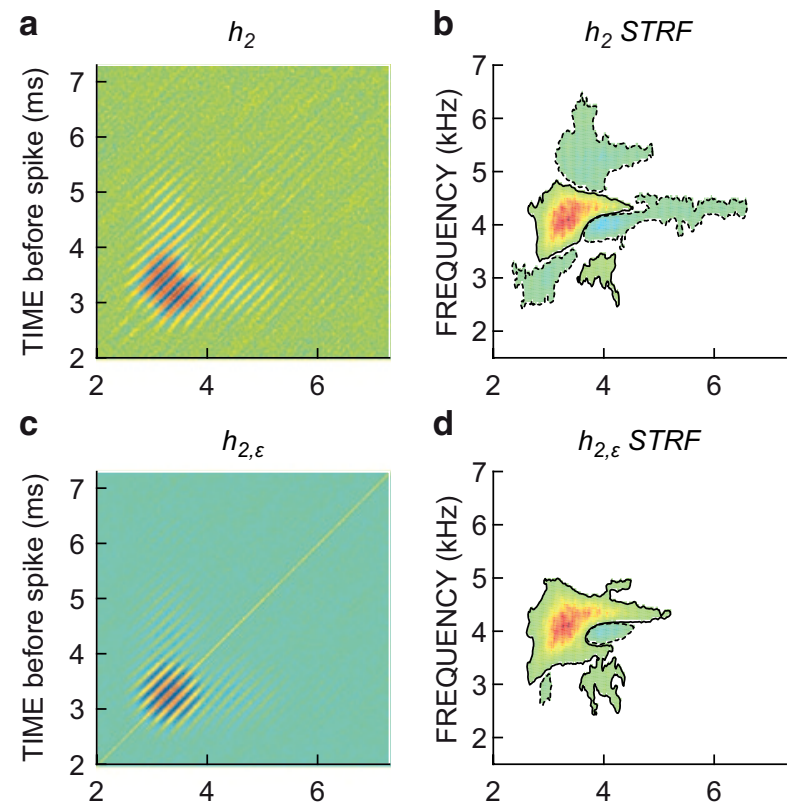

d
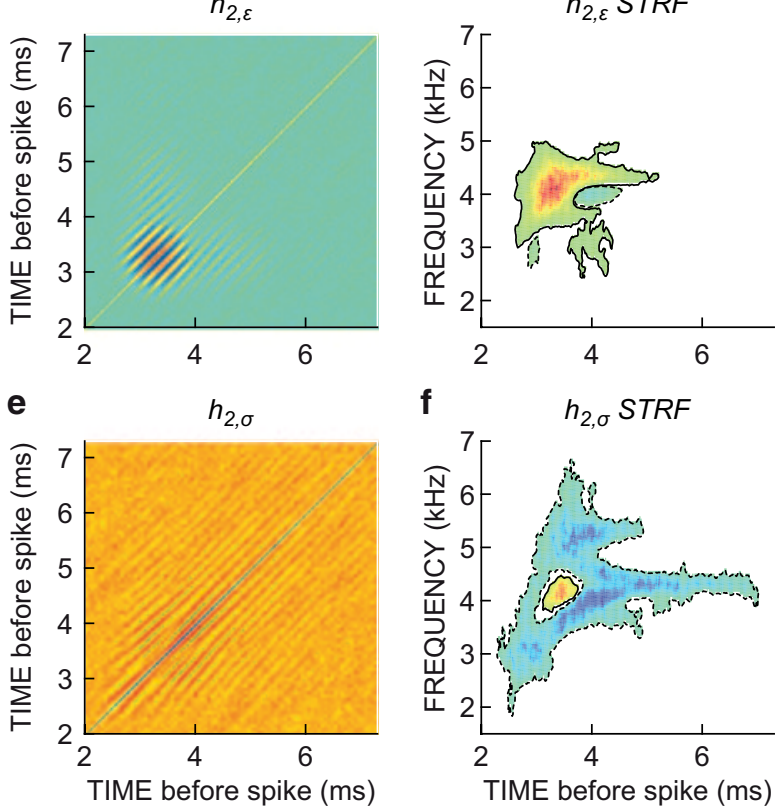

Fig. 4 Second-order Wiener-kernel, excitatory and suppressive sub-kernels, and their spectrotemporal receptive-field equivalents calculated from the responses of a single medium-spontaneous-rate ANF in a NH chinchilla: $\mathrm{CF}=4.2 \mathrm{kHz}, \theta=20$-dB SPL, $Q_{10} 3.6, \mathrm{SR}=6.0$ spikes s$^{-1}$. 21,161 driven spike times are included in the analysis. Right-hand column, warm colors and solid lines indicate areas of excitation, cool colors and dashed lines areas of suppression, with z-score $>3$ w.r.t. the bootstrap distribution 
pressive regions flanking the main excitatory region along the frequency axis, with a high-to-low frequency glide (early-to-late) consistent with travelling-wave delay (Fig. 4b). Decomposing $h_{2}$ into its excitatory and suppressive sub-kernels, we observe a broad area of suppressive tuning which overlaps with the excitatory tuning in time and frequency (Fig. 4d and f). There is also an "on-CF" component to the suppression, occurring before the excitation.

There is substantial variability in the relative contribution of suppression to ANF responses (Fig. 5a), similar to that observed with tone stimuli in the "fractionalresponse" metric (e.g., Miller et al. 1997). There is a group of fibers (both NH and $\mathrm{HI})$ across the $\mathrm{CF}$ axis, which show no significant suppression $\left(R_{\varepsilon, \sigma}=1\right.$; Fig. 5a). These are mainly, but not exclusively, of the high-spontaneous-rate class $(\geq 18$ spikes $\left.\mathrm{s}^{-1}\right)$. HI fibers in the region of greatest damage $(\sim 2-5 \mathrm{kHz})$ tend to have reduced suppression. Plotting $R_{\varepsilon, \sigma} v s$. CF-normalized 10-dB bandwidth, we find a significant correlation between broadened tuning and loss of suppression (Fig. 5b). Considering only $\mathrm{CFs}>2 \mathrm{kHz}$ in this linear regression increased the variance explained from 9.1 to $18.4 \%$.

\section{Discussion}

Using tones and broadband noise, we found significant changes in the pattern of suppression in the responses of ANFs following noise-induced hearing loss, likely reflecting outer-hair-cell disruption. Previous studies have examined the relationship between ANF 2TS and chronic low-level noise exposure coupled with ageing in the Mongolian gerbil (Schmiedt et al. 1990; Schmiedt and Schultz 1992), and following an acute intense noise exposure in the cat (Miller et al. 1997). Ageing in a noisy environment was associated with a loss of 2TS. Schmiedt and colleagues often found complete absence of high-side 2TS, with sparing of low-side 2TS, even in cochlear regions with up to $60 \%$ outer-hair-cell loss; suggesting potentially different mechanisms for low- and high-side suppression. Our 2TS data are in broad agreement with these earlier findings: HI chinchillas had elevated high-side 2TS thresholds, but retained sensitivity to low-side suppressors. Miller et al. (1997) related a reduction in 2TS in ANFs to changes in the representation of voiced vowel sounds. Weakened compressive nonlinearities contributed to a reduction in "synchrony capture" by stimulus harmonics near vowel-formant peaks. These effects likely contribute to deficits in across-CF spatio-temporal coding of temporal-finestructure information in speech for HI listeners (e.g., Heinz et al. 2010).

Although the 2TS approach provides important insights on cochlear nonlinearities, it is important to consider the effects of hearing impairment on cochlear nonlinearity in relation to broadband sounds. Our Wiener-kernel approach demonstrated reduced suppression in ANF responses from HI animals, which was correlated with a loss of frequency selectivity. These HI animals also exhibited reduced magnitude DPOAEs: additional evidence of reduced compressive nonlinearity. However, the analyses presented here (Fig. 5) only quantify the overall relative suppressive influ- 

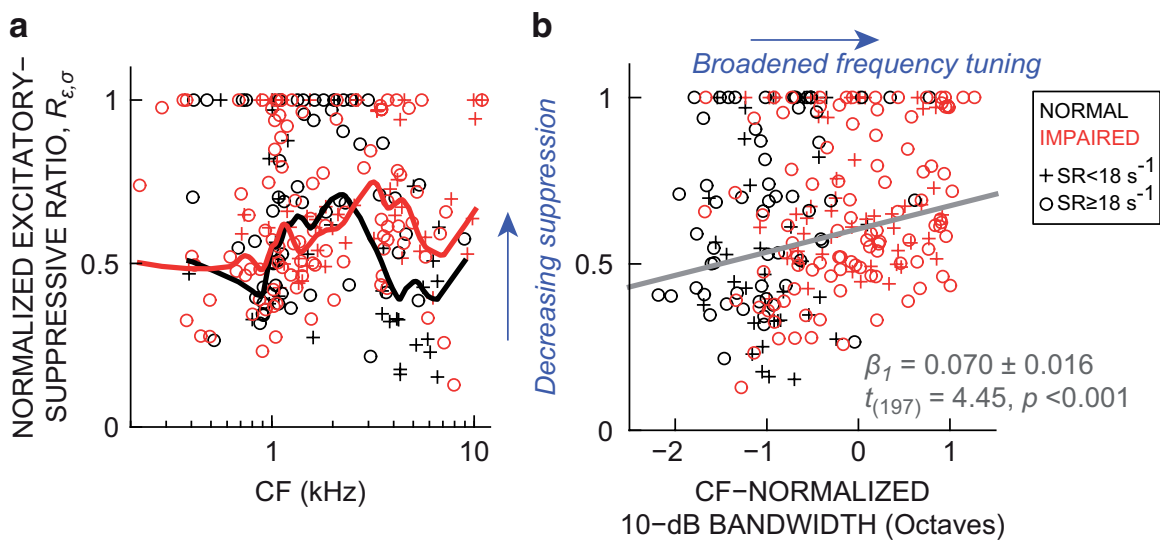

Fig. 5 Normalized excitatory-suppressive ratio $v s$. CF (a), and $v s$. CF-normalized 10-dB bandwidth (b). a, Solid lines, lowess fits to values of $R_{\varepsilon, \sigma}<1$. b. Horizontal axis expresses 10-dB bandwidth in octaves relative to the 95th percentile of NH-chinchilla ANF data (Kale and Heinz 2010). Gray line and text, least-squares linear fit to values of $R_{\mathcal{E}, \sigma}<1$

ence on ANF responses. Using the STRFs derived from $h_{2} \mathrm{~s}$, we aim to characterize the timing and frequency tuning of suppression in the HI auditory periphery in response to broadband sounds. Moreover, these same techniques can be exploited to address potential changes in the balance between excitation and inhibition ("central gain change") in brainstem nuclei following damage to the periphery. Such approaches have previously proved informative across sensory modalities (e.g., Rust et al. 2005), and will likely yield results with tangible translational value.

Acknowledgements Funded by NIH R01-DC009838 (M.G.H.) and Action on Hearing Loss (UK-US Fulbright Commission scholarship; M.S.). We thank Sushrut Kale, Kenneth S. Henry, and Jon Boley for re-use of ANF noise-response data, and Bertrand Fontaine and Kenneth S. Henry for helpful discussions on spike-triggered neural characterization.

Open Access This chapter is distributed under the terms of the Creative Commons AttributionNoncommercial 2.5 License (http://creativecommons.org/licenses/by-nc/2.5/) which permits any noncommercial use, distribution, and reproduction in any medium, provided the original author(s) and source are credited.

The images or other third party material in this chapter are included in the work's Creative Commons license, unless indicated otherwise in the credit line; if such material is not included in the work's Creative Commons license and the respective action is not permitted by statutory regulation, users will need to obtain permission from the license holder to duplicate, adapt or reproduce the material. 


\section{References}

Cooper NP (1996) Two-tone suppression in cochlear mechanics. J Acoust Soc Am 99(5):3087-3098

de Boer E, Nuttall AL (2002) The mechanical waveform of the basilar membrane. IV. Tone and noise stimuli. J Acoust Soc Am 111(2):979-989

Delgutte B (1990) Two-tone rate suppression in auditory-nerve fibers: dependence on suppressor frequency and level. Hear Res 49(1-3):225-246

Geisler CD, Yates GK, Patuzzi RB, Johnstone BM (1990) Saturation of outer hair cell receptor currents causes two-tone suppression. Hear Res 44(2-3):241-256

Heinz MG, Swaminathan J, Boley J, Kale S (2010) Across-fiber coding of temporal fine-structure: effects of noise-induced hearing loss on auditory nerve responses. In: Lopez-Poveda EA, Palmer AR, Meddis R (eds) The neurophysiological bases of auditory perception. Springer, New York, pp 621-630

Henry KS, Kale S, Scheidt RE, Heinz MG (2011) Auditory brainstem responses predict auditory nerve fiber thresholds and frequency selectivity in hearing impaired chinchillas. Hear Res 280(1-2):236-244

Hicks ML, Bacon SP (1999) Effects of aspirin on psychophysical measures of frequency selectivity, two-tone suppression, and growth of masking. J Acoust Soc Am 106(3):1436-1451

Kale S, Heinz MG (2010) Envelope coding in auditory nerve fibers following noise-induced hearing loss. J Assoc Res Otolaryngol 11(4):657-673. doi:10.1007/s10162-010-0223-6

Lewis ER, van Dijk P (2004) New variation on the derivation of spectro-temporal receptive fields for primary auditory afferent axons. Hear Res 189(1-2):120-136

Lewis ER, Henry KR, Yamada WM (2002a) Tuning and timing in the gerbil ear: wiener-kernel analysis. Hear Res 174(1-2):206-221

Lewis ER, Henry KR, Yamada WM (2002b) Tuning and timing of excitation and inhibition in primary auditory nerve fibers. Hear Res 171(1-2):13-31

Liberman MC (1984) Single-neuron labeling and chronic cochlear pathology. I. Threshold shift and characteristic-frequency shift. Hear Res 16(1):33-41

Miller RL, Schilling JR, Franck KR, Young ED (1997) Effects of acoustic trauma on the representation of the vowel "eh" in cat auditory nerve fibers. J Acoust Soc Am 101(6):3602-3616

Recio-Spinoso A, Temchin AN, van Dijk P, Fan YH, Ruggero MA (2005) Wiener-kernel analysis of responses to noise of chinchilla auditory-nerve fibers. J Neurophysiol 93(6):3615-3634

Rust NC, Schwartz O, Movshon JA, Simoncelli EP (2005) Spatiotemporal elements of macaque V1 receptive fields. Neuron 46(6):945-956

Sachs MB, Kiang NY (1968) Two-tone inhibition in auditory-nerve fibers. J Acoust Soc Am 43(5):1120-1128

Sachs MB, Young ED (1980) Effects of nonlinearities on speech encoding in the auditory nerve. J Acoust Soc Am 68(3):858-875

Schmiedt RA, Schultz BA (1992) Physiologic and histopathologic changes in quiet- and noiseaged gerbil cochleas. In: Dancer AL, Henderson D, Salvi RJ, Hamernik RP (eds) Noise-induced hearing loss. Mosby, St. Louis, pp 246-256

Schmiedt RA, Mills JH, Adams JC (1990) Tuning and suppression in auditory nerve fibers of aged gerbils raised in quiet or noise. Hear Res 45(3):221-236

Schwartz O, Pillow JW, Rust NC, Simoncelli EP (2006) Spike-triggered neural characterization. $\mathrm{J}$ Vis 6(4):484-507

Sneary MG, Lewis ER (2007) Tuning properties of turtle auditory nerve fibers: evidence for suppression and adaptation. Hear Res 228(1-2):22-30

Versteegh CP, van der Heijden M (2012) Basilar membrane responses to tones and tone complexes: nonlinear effects of stimulus intensity. J Assoc Res Otolaryngol 13(6):785-798. doi:10.1007/ s10162-012-0345-0

Versteegh CP, van der Heijden M (2013) The spatial buildup of compression and suppression in the mammalian cochlea. J Assoc Res Otolaryngol 14(4):523-545. doi:10.1007/s10162-013-0393-0 